Published in final edited form as:

Biochemistry. 2018 July 24; 57(29): 4241-4246. doi:10.1021/acs.biochem.8b00595.

\title{
D-amino acid-mediated translation arrest is modulated by the identity of the incoming aminoacyl-tRNA
}

\author{
Rachel C. Fleisher, Virginia W. Cornish, and Ruben L. Gonzalez Jr. ${ }^{*}$ \\ Department of Chemistry, Columbia University, New York, New York 10027, USA
}

\begin{abstract}
A complete understanding of the determinants that restrict $\mathrm{D}$-amino acid incorporation by the ribosome, which is of interest to both basic biologists as well as the protein engineering community, remains elusive. Previously, we demonstrated that D-amino acids are successfully incorporated into the $\mathrm{C}$-terminus of the nascent polypeptide chain. Ribosomes carrying the resulting peptidyl-D-aminoacyl-tRNA (peptidyl-D-aa-tRNA) donor substrate, however, partition into subpopulations that either undergo translation arrest through inactivation of the ribosomal peptidyl-transferase center (PTC) or remain translationally competent. The proportion of each subpopulation is determined by the identity of the D-amino acid sidechain. Here, we demonstrate that the identity of the aminoacyl-tRNA (aa-tRNA) acceptor substrate that is delivered to ribosomes carrying a peptidyl-D-aa-tRNA donor further modulates this partitioning. Our discovery demonstrates that it is the pairing of the peptidyl-D-aa-tRNA donor and the aa-tRNA acceptor that determines the activity of the PTC. Moreover, we provide evidence that both the amino acid and tRNA components of the aa-tRNA acceptor contribute synergistically to the extent of arrest. The results of this work deepen our understanding of the mechanism of D-amino acid-mediated translation arrest and how cells avoid this precarious obstacle, reveal similarities to other translation arrest mechanisms involving the PTC, and provide a new route for improving the yields of engineered proteins containing D-amino acids.
\end{abstract}

During protein synthesis, peptide bond formation is catalyzed by the ribosomal peptidyltransferase center (PTC). This reaction involves transfer of the nascent polypeptide chain from the peptidyl-tRNA donor in the ribosomal peptidyl-tRNA binding $(\mathrm{P})$ site to the aminoacyl-tRNA (aa-tRNA) acceptor in the ribosomal aa-tRNA binding (A) site (Fig. 1A). Although the PTC has evolved to catalyze peptide bond formation with a remarkable diversity of proteinogenic amino acids, these substrates are exclusively $\mathrm{L}$ enantiomers or prochiral. Nonetheless, D-amino acids can be found in the cell at high concentrations ${ }^{1,2}$.

\footnotetext{
*To whom correspondence should be addressed: Ruben L. Gonzalez Jr., Department of Chemistry, Columbia University, 3000 Broadway, MC3126, New York, NY 10027, USA, Tel.: (212) 854-1096, Fax: (212) 932-1289, rlg2118@ columbia.edu. Author Contributions

R.C.F. and R.L.G. designed the research; R.C.F. performed the experiments and analyzed the data; R.C.F. and R.L.G. interpreted the results. R.C.F. and R.L.G. wrote the manuscript; all three authors approved the final manuscript.

Competing Financial Interests

The authors declare no competing financial interests.

Supporting Information

The Supporting Information is available free of charge on the ACS Publications website. Materials and Methods, Figures S1-S2, Table S1.
} 
Moreover, it is well-established that at least some aa-tRNA synthetase enzymes are capable of aminoacylating D-amino acids onto tRNAs ${ }^{3,4}$, suggesting the possibility that the translation machinery (TM) may have to contend with D-aa-tRNAs in vivo. Consistent with this possibility, a D-aa-tRNA deacylase enzyme has been identified that is nearly universally conserved and serves to remove D-amino acids from the tRNAs onto which they have been misacylated ${ }^{5}$. Although these observations suggest that D-aa-tRNAs might present a challenge to the TM, how D-aa-tRNAs that are successfully delivered to the translating ribosome impact protein synthesis remains poorly understood, with reported D-amino acid incorporation efficiencies ranging anywhere from zero to $40 \%$ or higher ${ }^{4,6-10}$. Nonetheless, continued efforts to develop a complete mechanistic understanding of how D-aa-tRNAs impede translation and/or how the TM might be engineered to facilitate the incorporation of D-aa-tRNAs are important for elucidating the limits of basic biology and enabling new directions in protein engineering 6,11 .

Using a highly purified, Escherichia coli-based, in vitro translation system devoid of D-aatRNA deacylase to perform biochemical assays reporting on the major steps of the translation elongation cycle (Fig. 1A), we recently published a mechanistic study of Damino acid incorporation by the $\mathrm{TM}^{12}$. The results of this study demonstrated that, despite reported differences in the ability of D-aa-tRNA acceptors to form complexes with the GTP bound-form of elongation factor (EF)-Tu (i.e., EF-Tu(GTP)D-aa-tRNA complexes) ${ }^{8,13,14}$, D-aa-tRNA acceptors are delivered to the A site in an EF-Tu(GTP)-dependent manner and participate in peptide bond formation with yields mirroring those obtained from delivery of the corresponding L-aa-tRNA acceptors. Nonetheless, EF-G(GTP)-catalyzed translocation of the resulting peptidyl-D-aa-tRNA from the A- to $\mathrm{P}$ sites resulted in ribosomal elongation complexes (ECs) that partition into two subpopulations. One subpopulation is unable to undergo peptidyl transfer with the next L-aa-tRNA acceptor and becomes translationally arrested. The second subpopulation can undergo peptidyl transfer with the next L-aa-tRNA acceptor and remains translationally competent. Remarkably, the fraction of ECs that undergo translation arrest or remain translationally competent is dependent on the side chain of the D-amino acid, suggesting that the observed arrest arises from a D-amino acid side chain-dependent stabilization of an inactive conformation of the PTC. This conclusion was supported by chemical probing and molecular dynamics simulations indicating that the peptidyl-D-aa-tRNA donor stabilizes conformations of 23S ribosomal RNA nucleotides A2058, A2059, A2062, A2063, G2505, and U2506 that are different from those which the corresponding peptidyl-L-aa-tRNA donor stabilizes. These nucleotides comprise part of the ribosomal nascent polypeptide exit tunnel entrance (ETE), a region of the ribosome that evidence suggests is conformationally and functionally coupled to the PTC ${ }^{15}$.

A striking feature of the study described above is that several of the nucleotides we identified are also implicated in macrolide ${ }^{16}$-, chloramphenicol ${ }^{17}$-, and nascent polypeptidemediated translation arrest mechanisms ${ }^{18,19}$. Perhaps most interestingly, a growing number of studies are finding that the extent of arrest in these mechanisms is modulated by the identity of the peptidyl-aa-tRNA donor and aa-tRNA acceptor pair in the P- and A sites of the arrested $\mathrm{EC}^{20-22}$. Motivated by these studies, we hypothesized that such a 'pairing effect' may likewise modulate the extent of D-amino acid-mediated translation arrest. To test this hypothesis, we have investigated whether the identity of the aa-tRNA acceptor can 
modulate the fraction of ECs carrying a peptidyl-D-aa-tRNA donor that undergo translation arrest or remain translationally competent.

Using our previously described in vitro translation system and tripeptide synthesis assay ${ }^{12}$, we performed fMet-L-Lys- $X$ and fMet-D-Lys-X tripeptide synthesis reactions, where " $\mathrm{X}$ " stands for L-Phe, L-Arg, L-Glu, or L-Val (Supporting Materials and Methods and Figs. 1B and S1). We note that throughout the manuscript, 'fMet' always denotes the L stereoisomer; the stereochemistry of all other amino acids is called out explicitly.The choice of peptidyl-DLys-tRNA ${ }^{\text {Lys }}$ as the donor in these experiments was driven by our previous demonstration that the fraction of arrested ECs is closest to $50 \%$ when a peptidyl-D-Lys-tRNA ${ }^{\text {Lys }}$ donor is paired with a L-Phe-tRNA ${ }^{\text {Phe }}$ acceptor ${ }^{12}$. We therefore reasoned that using the peptidyl-DLys-tRNA ${ }^{\text {Lys }}$ donor and L-Phe-tRNA ${ }^{\text {Phe }}$ acceptor pairing as a reference would allow us to most easily detect both increases and decreases in the fraction of arrested ECs. The choice of L-Phe-tRNA $^{\text {Phe }}$, L-Arg-tRNA $^{\text {Arg }}$, L-Glu-tRNA $^{\text {Glu }}$, and L-Val-tRNA ${ }^{\text {Val }}$ as acceptors allowed us to test how D-amino acid-mediated translation arrest is affected by amino acid hydrophobicity (L-Phe), electrostatics (L-Arg and L-Glu), and beta-branching (L-Val). To validate our experimental system, we performed tripeptide synthesis reactions using L-PhetRNA $^{\text {Phe }}$ as the acceptor (Figs. 2 and S2). The yields of the resulting fMet-L-Lys-L-Phe and fMet-D-Lys-L-Phe tripeptides were $89 \pm 1 \%$ and $64 \pm 1 \%$, respectively, in agreement with our previous observations of $80 \pm 1 \%$ and $58 \pm 5 \%$, respectively ${ }^{12}$.

Subsequently, we performed tripeptide synthesis reactions using L-Arg-tRNA ${ }^{\text {Arg }}$ as the aatRNA acceptor (Figs. S2 and 2). In this case, the fMet-L-Lys-L-Arg and fMet-D-Lys-L-Arg tripeptide synthesis reactions exhibited yields of $83 \pm 1 \%$ and $5 \pm 2 \%$, respectively. The latter result stands in stark contrast to the yield of $64 \pm 1 \%$ observed in the fMet-D-Lys-LPhe tripeptide synthesis reaction, revealing that the fraction of arrested ECs depends on the identity of the aa-tRNA acceptor.

Given the striking effect that we observed when the aa-tRNA acceptor carried a positively charged amino acid, we used the next tripeptide synthesis reactions to test the performance of an aa-tRNA acceptor carrying a negatively charged amino acid, L-Glu-tRNA ${ }^{\text {Glu }}$ (Figs. S2 and 2).

Here, we observed yields of $92 \pm 1 \%$ and $19 \pm 2 \%$ for the synthesis of the fMet-L-Lys-LGlu and fMet-D-Lys-L-Glu tripeptides, respectively. The data we have obtained here using aa-tRNA acceptors carrying charged amino acids are notably similar to our previous findings in which delivery of an L-Lys-tRNA ${ }^{\text {Lys }}$ acceptor to an EC carrying either fMet-D-PhetRNA $^{\text {Phe }}$ or fMet-D-Val-tRNA ${ }^{\text {Val }}$ donors results in $18 \pm 3 \%$ and $11 \pm 1 \%$ of the ECs undergoing translation arrest, respectively ${ }^{12}$. Collectively, our results suggest that charged aa-tRNA acceptors exacerbate D-amino acid-mediated translation arrest.

We next performed tripeptide synthesis reactions in which L-Val-tRNA ${ }^{\text {Val }}$ served as the incoming aa-tRNA (Figs. S2 and 2). Surprisingly, the fMet-L-Lys-L-Val and fMet-D-Lys-LVal tripeptide synthesis reactions exhibited yields of $88 \pm 6 \%$ and $97 \pm 5 \%$, respectively. This result again highlights the ability of the aa-tRNA acceptor to modulate the extent of Damino acid-mediated translation arrest. Perhaps most interestingly, these findings 
demonstrate that, by judiciously choosing the identity of the aa-tRNA acceptor, it is possible to completely bypass $\mathrm{D}$-amino acid-mediated translation arrest and incorporate a D-amino acid into a ribosome-synthesized protein or polypeptide as effectively as the corresponding L-amino acid. While here we have demonstrated how a small, representative set of L-aatRNA acceptors can modulate the D-amino acid-mediated translation arrest caused by a single peptidyl-D-aa-tRNA donor, additional experiments using a larger set of both L-aatRNA acceptors and peptidyl-D-aa-tRNA donors will be needed to fully elucidate the rules that govern this pairing effect.

The ability of the aa-tRNA acceptor to modulate the extent of D-amino acid-mediated translation arrest can originate from either its amino acid- or tRNA component. In order to dissect the contributions of these components, we misacylated L-phenylalanine onto tRNA $^{\text {Glu }}$ to generate L-Phe-tRNA ${ }^{\text {Glu }}$ (Supporting Materials and Methods and Fig. S1) 12,23 and used it as the aa-tRNA acceptor in the next tripeptide synthesis reactions (Figs. S2 and 3). If the L-phenylalanine component is the major contributor, then we would expect an fMet-D-Lys-L-Phe tripeptide yield that is similar or identical to that obtained upon delivery of L-Phe-tRNA ${ }^{\mathrm{Phe}}$ (i.e., $64 \pm 1 \%$ ). In contrast, if the tRNA ${ }^{\mathrm{Glu}}$ component is the major contributor, then we would expect an fMet-D-Lys- L-Phe tripeptide yield that is similar or identical to that obtained upon delivery of L-Glu-tRNA ${ }^{\text {Glu }}$ (i.e., $19 \pm 2 \%$ ). Contrary to either expectation, fMet-L-Lys-L-Phe and fMet-D-Lys-L-Phe tripeptide synthesis reactions using L-Phe-tRNA ${ }^{\mathrm{Glu}}$ as the aa-tRNA acceptor exhibited yields of $95 \pm 3 \%$ and $95 \pm 2 \%$, respectively. These results demonstrate that both the amino acid and tRNA ${ }^{9,10}$ components of the aa-tRNA acceptor contribute to modulating the extent of D-amino acid-mediated translation arrest and do so synergistically. Nonetheless, it is clear that additional experiments using a more expansive set of misacylated aa-tRNA acceptors will be needed to fully comprehend the contributions that the amino acid- and tRNA components of the aatRNA acceptor make to modulating the extent of D-amino acid-mediated translation arrest.

In this communication, we demonstrate that the identity of the incoming aa-tRNA acceptor can modulate the fraction of ECs carrying a peptidyl-D-aa-tRNA donor that undergo Damino acid-mediated translation arrest. In addition, we show that the amino acid- and tRNA components of the incoming aa-tRNA contribute synergistically to modulating the extent of translation arrest. Extending our previously proposed model for D-amino acid-mediated translation arrest ${ }^{12}$, our data suggests that translocation of the peptidyl-D-aa-tRNA into the $\mathrm{P}$ site alters the conformational dynamics of the PTC. Specifically, the peptidyl-D-aa-tRNA likely limits the conformational space that the PTC can sample and, to an extent that depends on the identity of the D-amino acid side chain, predisposes the PTC to stably adopt an inactive conformation. The incoming aa-tRNA acceptor can then further modulate the dynamics of the PTC, biasing the PTC towards stably adopting an inactive conformation that induces translation arrest or an active conformation that enables continued translation. The ability of a particular aa-tRNA acceptor to bias the PTC towards stably adopting an inactive conformation establishes the fraction of arrested ECs. Thus, the extent of D-amino acidmediated translation arrest is not determined solely by the side chain of the D-amino acid, as we originally proposed, but rather by the identity of a particular peptidyl-D-aa-tRNA donor and aa-tRNA acceptor pair (Fig. 4). 
Previously, we have demonstrated that translocation of a peptidyl-D-aa-tRNA into the P site allosterically induces a conformational change of $23 \mathrm{~S}$ rRNA nucleotides that comprise the ETE. Intriguingly, several other translation arrest mechanisms involving these same ETE nucleotides likewise demonstrate a pairing effect similar to that described here. For example, in their studies of a nascent polypeptide-mediated translation arrest mechanism involving contacts of the nascent polypeptide with these ETE nucleotides ${ }^{24}$, Mankin and co-workers demonstrated that the extent to which they could exacerbate or relieve the corresponding nascent polypeptide-mediated translation arrest depended on the identity of the aa-tRNA acceptor $^{21}$. Surprisingly, they observed that L-Val-tRNA ${ }^{\mathrm{Val}}$ and L-Phe-tRNA ${ }^{\text {Phe }}$ acceptors exhibited among the lowest arrest efficiencies, while L-Arg-tRNA ${ }^{\text {Arg }}$ and L-Glu-tRNA Glu acceptors exhibited among the highest, a trend that is consistent with the trend that we have observed here. Mankin and co-workers have also shown that binding of a macrolide antibiotic to these ETE nucleotides robustly induces translation arrest primarily in the context of specific, problematic peptidyl-aa-tRNA donor and aa-tRNA acceptor pairs ${ }^{20}$. Similar conformational and pairing effects were observed in the context of the PTC-binding antibiotics chloramphenicol and linezolid ${ }^{16,22}$. Thus, our findings contribute to an emerging understanding of a conformational and functional link between the ETE and the PTC, in which translation arrest can be induced by particular peptidyl-aa-tRNA donor and aa-tRNA acceptor pairs.

The results we report here also demonstrate the potentially devastating effects that incorporating a $\mathrm{D}$-amino acid into the nascent polypeptide chain can have on translation, and highlight the important role that D-aa-tRNA deacylase enzymes play in protecting ECs under stress conditions in which D-amino acids may be misacylated onto tRNAs and delivered to $\mathrm{ECs}^{25}$. Similarly, recent work from Church and co-workers has shown that translation arrest resulting from incorporation of consecutive D-amino acids can be relieved by EF-P 26 , a regulatory translation factor that has been shown to rescue ribosomes that have become arrested while synthesizing polyproline motifs ${ }^{27,28}$, translating mRNA sequences prone to +1 frameshifting ${ }^{29}$, and translocating peptidyl-tRNAs produced with miscoded aatRNAs ${ }^{30}$. Whether EF-P or other cellular factors that function to rescue ribosomes that have become translationally arrested ${ }^{31}$ can also relieve D-amino acid-mediated translation arrest, either in vitro or in vivo, remains to be investigated. Furthermore, the response of the eukaryotic $\mathrm{TM}^{32}$ to D-aa-tRNAs is currently unknown and it is possible that D-aa-tRNAs might affect the eukaryotic TM in a manner that is different from how they affect the bacterial $\mathrm{TM}^{31}$. Thus, further studies are needed to fully understand the scope, extent, and control of D-amino acid-mediated translation arrest.

Finally, our results reveal a straightforward approach for improving the efficiency with which D-amino acids can be incorporated into proteins or polypeptides for protein engineering applications. By simply optimizing the pairing of peptidyl-D-aa-tRNA donors and aa-tRNA acceptors, the efficiency of incorporation can be improved dramatically without the need for complex engineering of the TM.

\section{Supplementary Material}

Refer to Web version on PubMed Central for supplementary material. 


\section{Acknowledgements}

We thank Dr. Colin Kinz-Thompson for his assistance in data analysis. R.C.F was supported by the Training Program in Molecular Biophysics at Columbia University (T32 GM008281). This work was supported in part by funds to V.W.C. and R.L.G. from Columbia University as well as National Institutes of Health (NIH) Grant R01 GM090126 (to R.L.G. and V.W.C.) and R01 GM119386 (to R.L.G.).

\section{REFERENCES}

(1). Cava F, Lam H, de Pedro MA, and Waldor MK (2011) Emerging knowledge of regulatory roles of D-amino acids in bacteria. Cell. Mol. Life Sci 68, 817-831. [PubMed: 21161322]

(2). Kiriyama Y, and Nochi H (2016) D-Amino Acids in the Nervous and Endocrine Systems. Scientifica (Cairo). 2016.

(3). Soutourina J, Plateau P, and Blanquet S (2000) Metabolism of D-aminoacyl-tRNAs in Escherichia coli and Saccharomyces cerevisiae cells. J. Biol. Chem 275, 32535-32542. [PubMed: 10918062]

(4). Calendar R, and Berg P (1967) D-Tyrosyl RNA: Formation, hydrolysis and utilization for protein synthesis. J. Mol. Biol 26, 39-54. [PubMed: 4292198]

(5). Wydau S, van der Rest G, Aubard C, Plateau P, and Blanquet S (2009) Widespread distribution of cell defense against D-aminoacyl-tRNAs. J. Biol. Chem 284, 14096-14104. [PubMed: 19332551]

(6). Tan Z, Forster AC, Blacklow SC, and Cornish VW (2004) Amino acid backbone specificity of the Escherichia coli translation machinery. J. Am. Chem. Soc 126, 12752-3. [PubMed: 15469251]

(7). Noren CJ, Anthony-Cahill SJ, Griffith MC, and Schultz PG (1989) A General Method for SiteSpecific Incorporation of Unnatural Amino Acids into Proteins 244, 182-188.

(8). Fujino T, Goto Y, Suga H, H. M. (2013) Reevaluation of the D-amino acid compatibility with the elongation event in translation. J. Am. Chem. Soc 135, 1830. [PubMed: 23301668]

(9). Katoh T, Tajima K, and Suga H (2017) Consecutive Elongation of D-Amino Acids in Translation. Cell Chem. Biol 24, 46-54. [PubMed: 28042044]

(10). Katoh T, Iwane Y, and Suga H (2017) Logical engineering of D-arm and T-stem of tRNA that enhances D-amino acid incorporation. Nucleic Acids Res 45, 12601-12610. [PubMed: 29155943]

(11). Ellman, Jonathan A, Mendel, David, Schultz PG (1992) Site-Specific Incorporation of Novel Backbone Structures into Proteins. Science (80-. ). 255, 197-200.

(12). Englander MT, Avins JL, Fleisher RC, Liu B, Effraim PR, Wang J, Schulten K, Leyh TS, Gonzalez RL, and Cornish VW (2015) The ribosome can discriminate the chirality of amino acids within its peptidyl-transferase center. Proc. Natl. Acad. Sci 112, 6038-6043. [PubMed: 25918365]

(13). Yamane T, Miller DL, and Hopfield J (1981) Discrimination between D- and L-tyrosyl transfer ribonucleic acids in peptide chain elongation. Biochemistry 20, 7059-7064. [PubMed: 7032588]

(14). Achenbach J, Jahnz M, Bethge L, Paal K, Jung M, Schuster M, Albrecht R, Jarosch F, Nierhaus $\mathrm{KH}$, and Klussmann S (2015) Outwitting EF-Tu and the ribosome: Translation with D-amino acids. Nucleic Acids Res 43, 5687-5698. [PubMed: 26026160]

(15). Sothiselvam S, Liu B, Han W, Ramu H, Klepacki D, Atkinson GC, Brauer A, Remm M, Tenson T, Schulten K, Vázquez-Laslop N, and Mankin AS (2014) Macrolide antibiotics allosterically predispose the ribosome for translation arrest. Proc. Natl. Acad. Sci. U. S. A 111, 9804-9809. [PubMed: 24961372]

(16). Moazed D, and Noller HF (1987) Chloramphenicol, erythromycin, carbomycin and vernamycin B protect overlapping sites in the peptidyl transferase region of $23 \mathrm{~S}$ ribosomal RNA. Biochimie 69, 879-884. [PubMed: 3122849]

(17). Ettayebi M, Prasad SM, and Morgan E. a. (1985) Chloramphenicol-erythromycin resistance mutations in a 23S rRNA gene of Escherichia coli. J Bacteriol 162, 551-557. [PubMed: 3886627]

(18). Nakatogawa H, and Ito K (2002) The ribosomal exit tunnel functions as a discriminating gate. Cell 108, 629-636. [PubMed: 11893334] 
(19). Yang R, Cruz-Vera LR, and Yanofsky C (2009) 23S rRNA nucleotides in the peptidyl transferase center are essential for tryptophanase operon induction. J. Bacteriol 191, 3445-3450. [PubMed: 19329641]

(20). Kannan K, Kanabar P, Schryer D, Florin T, Oh E, Bahroos N, Tenson T, Weissman JS, and Mankin AS (2014) The general mode of translation inhibition by macrolide antibiotics. Proc. Natl. Acad. Sci. U. S. A 111, 1-6.

(21). Ramu H, Vázquez-Laslop N, Klepacki D, Dai Q, Piccirilli J, Micura R, and Mankin AS (2011) Nascent Peptide in the Ribosome Exit Tunnel Affects Functional Properties of the A-Site of the Peptidyl Transferase Center. Mol. Cell 41, 321-330. [PubMed: 21292164]

(22). Marks J, Kannan K, Roncase EJ, Klepacki D, Kefi A, Orelle C, Vázquez-Laslop N, and Mankin AS (2016) Context-specific inhibition of translation by ribosomal antibiotics targeting the peptidyl transferase center. Proc. Natl. Acad. Sci. U. S. A 113, 12150-12155. [PubMed: 27791002]

(23). Murakami H, Ohta A, Ashigai H, and Suga H (2006) A highly flexible tRNA acylation method for non-natural polypeptide synthesis. Nat. Methods 3, 357-359. [PubMed: 16628205]

(24). Ramu H, Mankin A, and Vazquez-Laslop N (2009) Programmed drug-dependent ribosome stalling: MicroReview. Mol. Microbiol 71, 811-824. [PubMed: 19170872]

(25). Soutourina O, Soutourina J, Blanquet S, and Plateau P (2004) Formation of D-tyrosyl-tRNATyr accounts for the toxicity of D-tyrosine toward Escherichia coli. J. Biol. Chem 279, 42560-42565. [PubMed: 15292242]

(26). Huang P, Wang F, Narasimhan K, Chatman K, Aach J, Sunia A, Spoering R, and Church GM (2017) Toward D-peptide biosynthesis: Elongation Factor P enables ribosomal incorporation of consecutive D-amino acids. bioRxiv.

(27). Doerfel LK, Wohlgemuth I, Kothe C, Peske F, Urlaub H, and Rodnina MV (2013) EF-P is essential for rapid synthesis of proteins containing consecutive proline residues. Science (80-. ). 339, 85-88.

(28). Peil L, Starosta AL, Lassak J, Atkinson GC, Virumäe K, Spitzer M, Tenson T, Jung K, Remme J, and Wilson DN (2013) Distinct XPPX sequence motifs induce ribosome stalling, which is rescued by the translation elongation factor EF-P. Proc. Natl. Acad. Sci. U. S. A 110, 15265-70. [PubMed: 24003132]

(29). Gamper HB, Masuda I, Frenkel-Morgenstern M, and Hou Y-M (2015) Maintenance of protein synthesis reading frame by EF-P and m1G37-tRNA. Nat. Commun 6, 7226. [PubMed: 26009254]

(30. ) Alejo JL, and Blanchard SC (2017) Miscoding-induced stalling of substrate translocation on the bacterial ribosome. Proc. Natl. Acad. Sci 201707539.

(31). Buskirk AR, and Green R (2017) Ribosome pausing, arrest and rescue in bacteria and eukaryotes. Philos. Trans. R. Soc. B Biol. Sci 372.

(32). Schuller AP, Wu CCC, Dever TE, Buskirk AR, and Green R (2017) eIF5A Functions Globally in Translation Elongation and Termination. Mol. Cell 66, 194-205.e5. [PubMed: 28392174] 
A

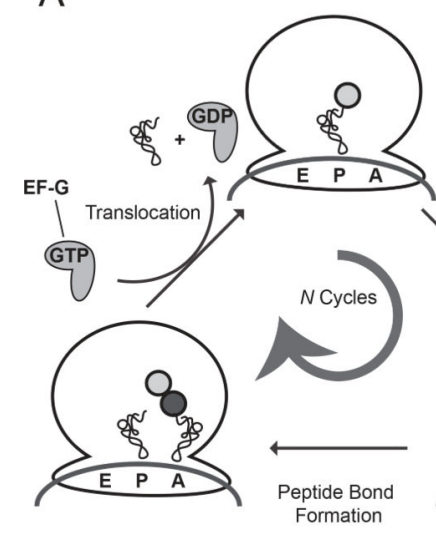

$\mathrm{B}$

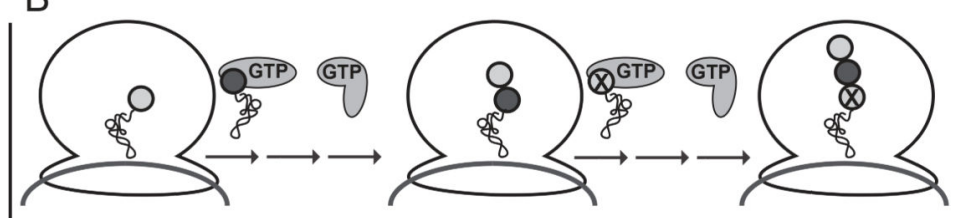

aa-tRNA Selection GDP

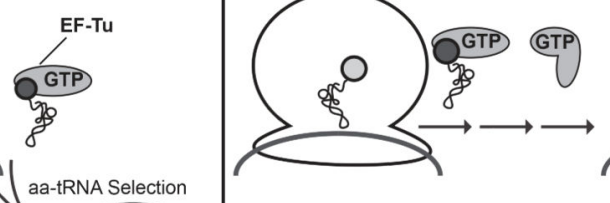

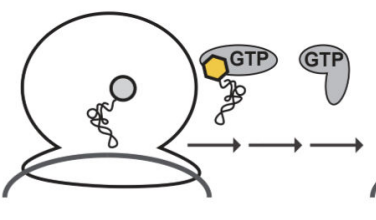

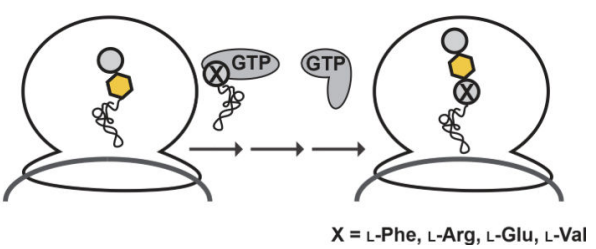

Figure 1. The experimental framework used to examine the role of the incoming aa-tRNA acceptor in D-amino acid-mediated translation arrest.

(A) The translation elongation cycle begins with delivery of EF-Tu(GTP)aa-tRNA complexes to an EC carrying a peptidyl-tRNA donor at the P site. Following selection of the correct, mRNA-encoded EF-Tu(GTP)aa-tRNA, GTP is hydrolyzed, EF-Tu(GDP) dissociates from the aa-tRNA and the EC, and the aa-tRNA is accommodated into the A site. Upon accommodation, peptide bond formation results in transfer of the nascent polypeptide chain from the peptidyl-tRNA donor at the $\mathrm{P}$ site to the aa-tRNA acceptor at the A site.

Subsequently, EF-G(GTP) catalyzes translocation of the P- and A-site tRNAs into the ribosomal tRNA exit (E) and P sites, with concomitant movement of the mRNA by one codon. (B) Tripeptide synthesis reactions were performed by delivering a mixture of either EF-Tu(GTP)L-Lys-tRNA ${ }^{\text {Lys }}$ (top row) or EF-Tu(GTP)D-Lys-tRNA ${ }^{\text {Lys }}$ (bottom row), EF$\mathrm{Tu}(\mathrm{GTP}) \mathrm{L}-\mathrm{X}$-tRNA ${ }^{\mathrm{X}}$, and EF-G(GTP) to ribosomal initiation complexes programmed with an mRNA encoding an fMet-Lys-X tripeptide (Table S1) and carrying a P-site f- $\left.{ }^{35} \mathrm{~S}\right]-$ MettRNA $^{\text {fMet }}$ (Supporting Materials and Methods). 

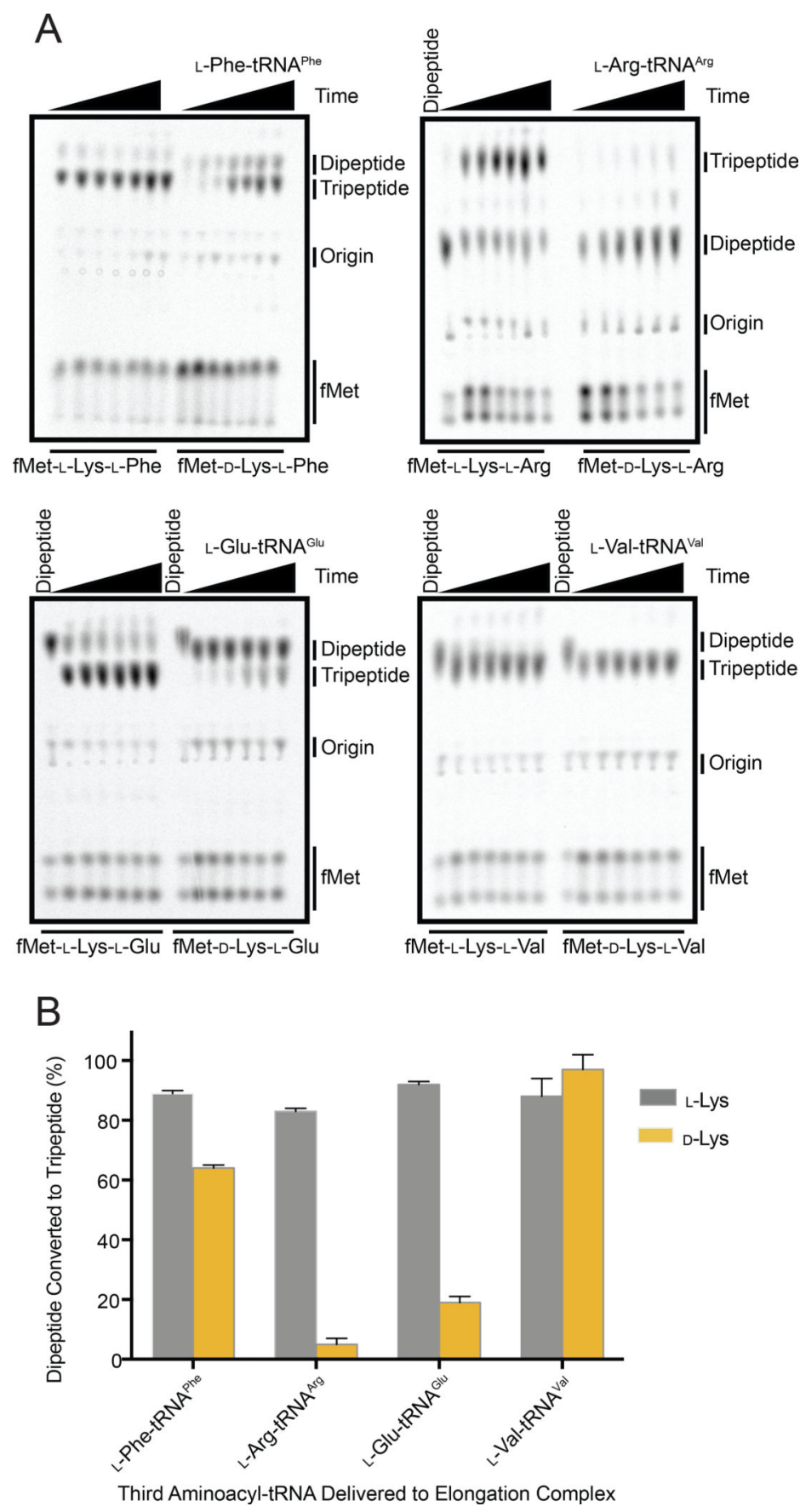

Figure 2. The identity of the incoming aa-tRNA acceptor modulates the extent of D-amino acidmediated translation arrest.

(A) Tripeptide synthesis reaction products were collected at reaction time points spanning 0$60 \mathrm{~min}$ in order to ensure that the reactions had gone to completion and we were measuring true end-point yields. Products were separated using electrophoretic thin layer chromatography (eTLC) and visualized by phosporimaging (Supporting Materials and Methods). (B) Yields of tripeptide synthesis reactions. Reaction time courses were performed in duplicate and in all cases were observed to go to completion by $30 \mathrm{~min}$ (Fig. $\mathrm{S} 2$ ). Consequently, the duplicate yield measurements collected at the 30 and 60 min time 
points for each tripeptide synthesis reaction were clustered, averaged, and reported as the mean reaction yield. Error bars represent the standard deviation of the mean (Supporting Materials and Methods). 

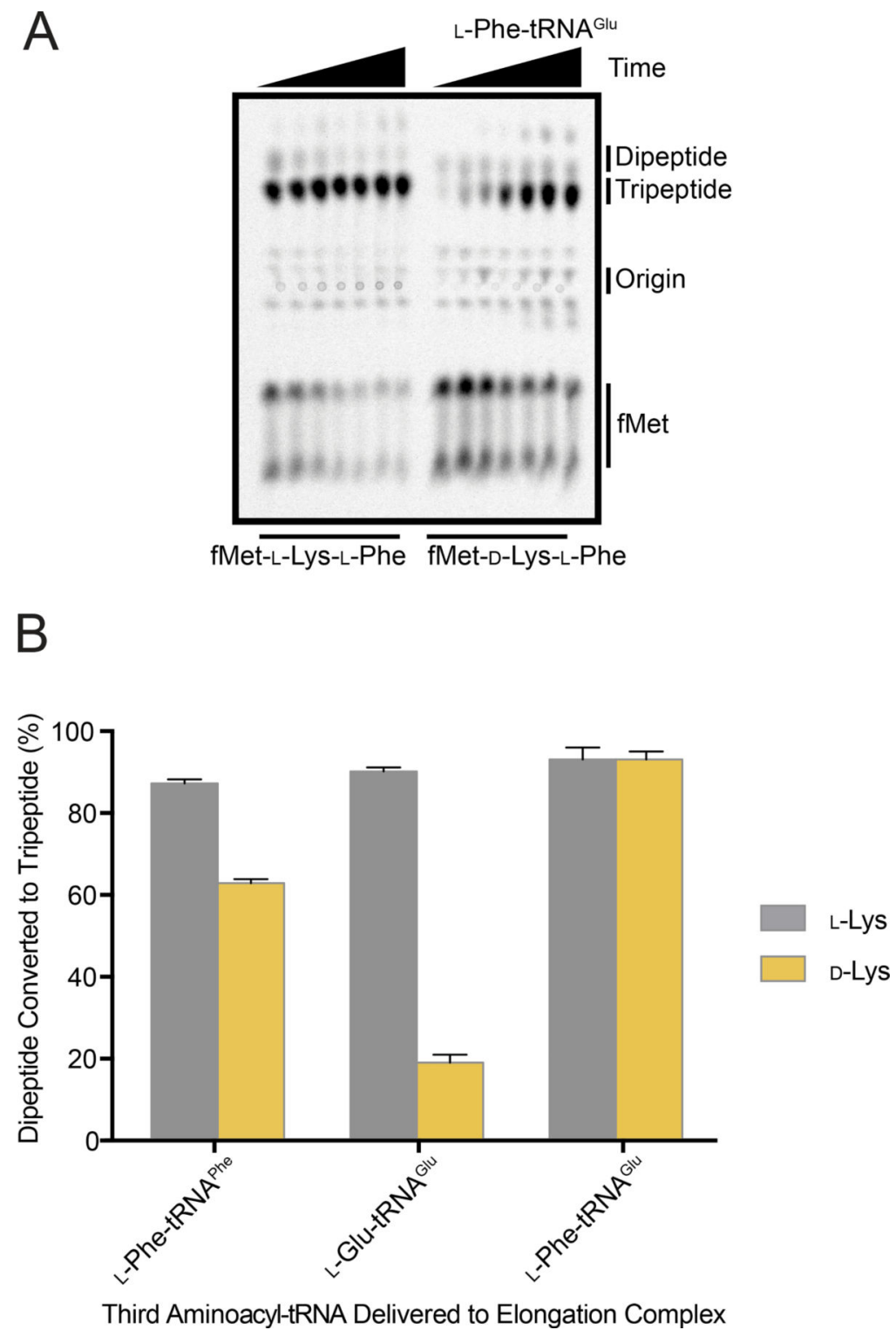

Figure 3. The amino acid- and tRNA components of the aa-tRNA acceptor contribute synergistically to the extent of $D$-amino acid-mediated translation arrest.

(A) eTLC of the fMet-L-Lys-L-Phe and fMet-D-Lys-L-Phe tripeptide synthesis reactions performed using L-Phe-tRNA ${ }^{\text {Glu }}$ as the aa-tRNA acceptor. (B) Yields of tripeptide synthesis reactions. Reaction time courses were performed in duplicate and in all cases were observed to go to completion by $30 \mathrm{~min}$ (Fig. S2). Consequently, the duplicate yield measurements collected at the 30 and $60 \mathrm{~min}$ time points for each tripeptide synthesis reaction were clustered, averaged, and reported as the mean reaction yield. Error bars represent the standard deviation of the mean (Supporting Materials and Methods). 

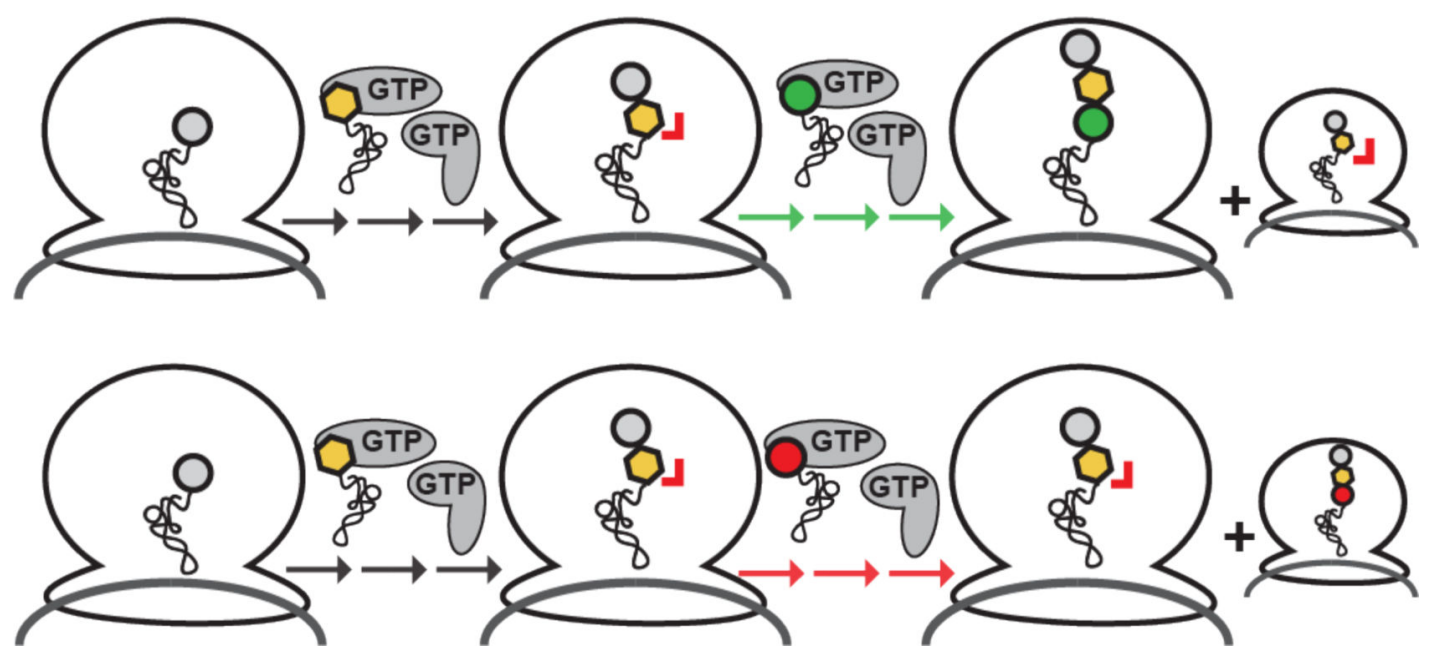

Figure 4. Extended mechanistic model for D-amino acid-mediated translation arrest. Translocation of a peptidyl-D-aa-tRNA donor into the P site predisposes the PTC to stably adopt an inactive conformation. Pairing of a particular peptidyl-D-aa-tRNA donor with an aa-tRNA acceptor that facilitates the ability of the PTC to stably adopt an inactive conformation results in a large fraction of arrested ECs (red aa-tRNA acceptor and pathway), whereas pairing with an aa-tRNA acceptor that interferes with the ability of the PTC to stably adopt an inactive conformation results in a large fraction of ECs remaining translationally competent (green aa-tRNA acceptor and pathway). 